\section{The multifaceted role of podoplanin expression in hepatocellular carcinoma}

\author{
Andreea Cioca, ${ }^{1}$ \\ Amalia R. Ceausu, ${ }^{2}$ Irina Marin, ${ }^{3}$ \\ Marius Raica, ${ }^{2}$ Anca M. Cimpean ${ }^{2}$ \\ ${ }^{1}$ Department of Patology, "Iuliu \\ Hatieganu" University of Medicine and \\ Pharmacy, Cluj-Napoca, Romania \\ ${ }^{2}$ Department of Microscopic \\ Morphology/Histology, Angiogenesis \\ Research Center, "Victor Babes" \\ University of Medicine and Pharmacy, \\ Timisoara, Romania \\ ${ }^{3}$ Department of Pathology, Sheba \\ Medical Center, Tel-Hashomer, Israel
}

\begin{abstract}
The role of podoplanin in hepatocellular carcinoma (HCC) is not clear yet. The aim of our study was to evaluate the expression of podoplanin in HCC and to determine its role in hepatocarcinogenesis. We performed immunohistochemistry with monoclonal D2-40 antibody, on paraffin-embedded tissue sections of 72 patients diagnosed with HCC. Lymphatic vessels density (LVD) was increased in patients who had vascular invasion at the time of diagnosis $(\mathrm{P}=0.018)$ and in those with associated cirrhosis $(\mathrm{P}=0.006)$. Tumor cells showing podoplanin expression were correlated with histological grade $(\mathrm{P}=0.040)$. Podoplanin-expressing cancer associated fibroblasts (CAFs) were correlated with both LVD $(\mathrm{P}=0.019)$ and tumor cells $(\mathrm{P}=0.015)$. Our results sustain the dual role of podoplanin in HCC by its involvement in both HCC tumorigenesis, lymphatic neovascularization and tumor invasion invasiveness. A possible crosstalk between epithelial and stromal tumor cells in $\mathrm{HCC}$ tumor microenvironment may be mediated by podoplanin, but this hypothesis needs further studies to elucidate this interrelation.
\end{abstract}

\section{Introduction}

Hepatocellular carcinoma (HCC) is one of the most frequent cancers worldwide. Despite the intense efforts in understanding the mechanisms and finding a cure, HCC remains one of the leading causes of worldwide cancer-related deaths. ${ }^{1}$ The treatment options in HCC are limited and finding new therapeutic targets is imperative.
Podoplanin is a 162 -amino acid transmembrane sialoglycoprotein that belongs to the family of type- 1 transmembrane sialomucin-like glycoproteins. ${ }^{2}$ It is expressed in various normal human tissues such as lymphatic endothelium, heart, lung, placenta, skeletal muscle, glomerular podocytes, mesothelial cells, Schwann cells, osteoblast, follicular dendritic cells, reticular fibroblasts, myofibroblasts and myoepithelial cells..$^{3-7}$ The monoclonal antibody D2-40 can detect podoplanin and currently is the most used antibody to demonstrate lymphatic endothelium. In addition to staining lymphatics, podoplanin was found to be positive in a relatively large spectrum of cancers including angiosarcomas, mesotheliomas, follicular dendritic cell sarcomas, testicular tumors, squamous cell carcinoma, thymomas and some subtypes of the central nervous system tumors. ${ }^{8-13}$ Also, podoplanin positivity was identified in CAFs of various malignancies and it seems that its expression in these cells plays an important role in tumor progression. ${ }^{14}$

The purpose of our study was to evaluate LVD in a series of human hepatocellular carcinoma cases by using monoclonal antibody against podoplanin (clone D2-40). In addition, we analyzed CAFs and tumor cells D2-40 immunoreactivity, in order to determine their role in hepatocarcinogenesis.

\section{Materials and Methods}

\section{Patients and tissue samples}

Our study included a total number of 72 patients, diagnosed with HCC, between 2002-2014, who underwent partial hepatectomy in the Department of Surgery of the "Prof. Dr. Octavian Fodor" Regional Institute of Gastroenterology and Hepatology, Cluj-Napoca, Romania. Tumor samples, as well as clinical data, were obtained from the Institute database according to protocols approved by the Ethical Committee and protocols followed the guidelines by the Helsinki Declaration. Surgically resected samples were fixed in $10 \%$ buffered formalin for $48 \mathrm{~h}$ and then they were embedded in paraffin followed the routine automated flow of this procedure. One section from each case was stained with haematoxylin and eosin for histopathology. Based on preliminary microscopic evaluation given by two independent pathologists for diagnosis, slides for immunohistochemistry were selected for each case.
Correspondence: Prof. Anca Maria Cimpean, Department of Microscopic Morphology/ Histology, Angiogenesis Research Center, "Victor Babes" University of Medicine and Pharmacy, Piata Eftimie Murgu 2, 300041 Timișoara, Romania. Tel: +40.720060955 .

E-mail: ancacimpean1972@yahoo.com

Key words: Hepatocellular carcinoma; podoplanin; lymphatic vessel density; cancer associated fibroblasts.

Received for publication: 29 July 2016. Accepted for publication: 3 February 2017.

This work is licensed under a Creative Commons Attribution-NonCommercial 4.0 International License (CC BY-NC 4.0).

(C) Copyright A. Cioca et al., 2017

Licensee PAGEPress, Italy

European Journal of Histochemistry 2017; 61:2707 doi:10.4081/ejh.2017.2707

\section{Clinicopathologic features}

Our study included samples from 72 patients, composed of 50 males $(70 \%)$ and 22 females (30\%), aged between 30-91 years (mean 64.13 years). In relation to tumor size, HCCs were divided into 2 categories: tumors smaller than $5 \mathrm{~cm}$ and tumors greater than $5 \mathrm{~cm}$. Of the $72 \mathrm{HCCs}$ included in this study, 28 tumors $(39 \%)$ were larger than $5 \mathrm{~cm}$ and $44(61 \%)$ were smaller than $5 \mathrm{~cm}$. According to Edmondson and Steiner system, tumor grade was divided into three groups: well differentiated (grade I- 8 cases), moderately differentiated (grade II-30 cases) and poorly differentiated (grades III and IV-34 cases). Tumor stages were classified according to TMN classification of tumors of the liver as follows: I (23 cases), II (26 cases), III (16 cases), and IV ( 7 cases). Among the 72 HCCs, cirrhosis was present in 32 cases (44\%), including hepatitis B in 12 cases and hepatitis $\mathrm{C}$ in 7 cases. Vascular invasion was noted in 25 cases (35\%).

\section{Immunohistochemistry}

Three- $\mu \mathrm{m}$ thick sections were cut from each case. Quality control of the specimens selected for immunohistochemistry was performed by applying immunostaining for vimentin clone V9 (Dako Agilent Technology, Carpinteria, CA, USA). Absence of vimentin immunoreaction was considered as an exclusion criterion. All immunohistochemical steps were performed by using BOND MAX Autostainer system with rigorously controlled steps from dewax to nuclear counterstain by settings of proper pre-designed program from 
the menu. For our purpose, we selected a program including dewax, hydrogen peroxide inhibition, $30 \mathrm{~min}$ incubation time for primary antibodies and we used a working system based on Bond Polymer Refine Detection System Brown Kit (Leica, Microsystems, Newcastle, UK), a standardized HRP free kit for automated immunohistochemistry including nuclear counterstain step. There were selected as primary antibodies anti human podoplanin antibodies (clone D2-40, Dako Agilent Technology) Slides removed from the Autostainer were automatically coverslipped by using Leica CV5030 Fully Automated Glass Coverslipper (Leica).

Image acquisition and data analysis were performed using Nikon Eclipse E 600 microscope (observed by using 200x and 400x magnification) and Lucia G software for microscopic image analysis.

\section{Evaluation of D2-40 staining}

The lymphatic microvascular density (LVD) was quantified intratumoral and peritumoral, by counting the podoplanin-positive vessels in three 'vascular hot spots', at high magnification (x200). 'Hot spot' areas were selected and defined as microscopic fields of 200x magnification containing the highest number of D2-40 positive lymphatic vessels. In these areas (three for each case) we counted D2-40 positive structures having a lymphatic vessels morphology with large lumen lined by D2-40 positive endothelial cells. In addition, we evaluated the intensity of podoplanin in tumor cells and in stroma cancer-associated fibroblasts (CAFs). The score was quantified on a scale of $1-3+$ as follows: low $(+)$, moderate $(++)$ or intense $(+++)$. The final score was assessed based on two criteria: intensity of the immunostaining and percent of positive cells. For this purpose, we used the morphometry software (NIS-Elements D 2.30, Laboratory Imaging s.r.o., Prague, Czech Republic) and a method previously described by Suciu et al., ${ }^{15}$ modified for cytoplasmic expression. Briefly, the software allowed us to apply a semi-automated method able to differentially assess positive and negative podoplanin expressing cells by detecting different intensities and counting positive podoplanin expressing cells. This method was used for tumor cells. The reaction to podoplanin was scored as follows: 0 (negative), absent or weak present in $<10 \%$ of tumor cells; $1+$ (low), weak in $>10 \%$ of tumor cells; $2+$ (moderate), weak or high expression in $>10 \%$ of tumor cells but less than $\leq 30 \%$ of tumor cells; and $3+$ (intense), high expression in $>30 \%$ of tumor cells.

\section{Statistical analysis}

Statistical analysis was performed to determine the relationship between podoplanin expression and the clinicopathologic parameters such as gender, age, presence of cirrhosis, chronic hepatitis, tumor stage, histological grading, tumor size and vascular invasion using Spearman's test. P-values of less than 0.05 values were considered statistically significant. All statistical analysis was performed using the SPSS 22.0 software.

\section{Results}

\section{Immunohistochemical findings}

Of 72 HCCs analyzed for lymphatic vessels, 32 (44.4\%) showed podoplaninpositive vessels within the intratumoral tissue, while 57 (79\%) revealed podoplaninpositive vessels within the peritumoral tissue (Figure 1A). Podoplanin positivity revealed that LVD was greater in peritumoral tissue (mean 14.6) compared with intratumoral tissue (mean 5.7). LVD was significantly related to background cirrhosis $(\mathrm{P}=0.006)$. We found that LVD was increased in patients who had vascular invasion at the time of diagnosis $(\mathrm{P}=0.018)$. Relationship between LVD and clinicopathological data are shown in Table 1.

We observed podoplanin expression in stromal spindle cells that were morphologically and immunohistochemically (vimentin positive) identified as CAFs (Figure 1B). CAFs were positive for podoplanin in 41 cases $(57 \%)$ and were correlated with both LVD $(\mathrm{P}=0.019)$ and podoplanin-expressing tumor cells $(\mathrm{P}=0.015)$. Moreover, examination of tumor cells showed cytoplasmatic positivity for podoplanin (Figure $1 \mathrm{C}, \mathrm{D}$ ) in 23 HCCs (32\%) including low expression in 14 cases $(60 \%)$, moderate expression in 7 cases $(31 \%)$, and intense expression in two cases $(9 \%)$. Podoplanin-positive cells were significantly correlated with the histological grade $(\mathrm{P}=0.040)$. Relationship between podoplanin expression by tumor cells and clinicopathological parameters are shown in Table 2

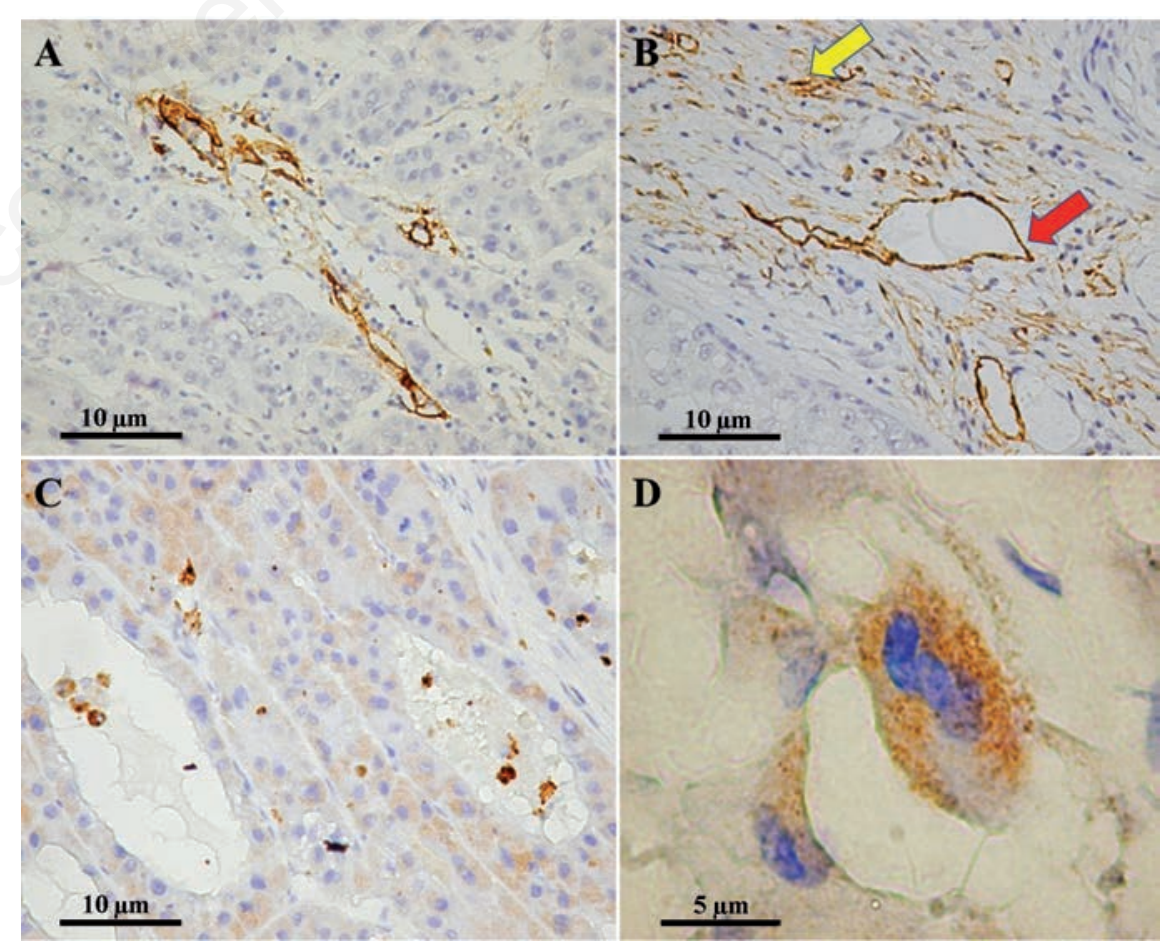

Figure 1. Podoplanin expression in human hepatocellular carcinoma showing: A) Positivity for lymphatic vessels and its absence in tumor cells; B) Positivity for cancer associated fibroblast (CAFs, yellow arrow) and stromal lymphatics (red arrow); C) Podoplanin cytoplasmatic staining of tumor cells at low magnification; D) Podoplanin cytoplasmatic staining at high magnification. 


\section{Discussion}

Tumor associated-lymphangiogenesis was identified as an important factor in the development and progression of various human cancers. ${ }^{16-19}$ Moreover, some preclinical experimental studies showed that inhibition of lymphangiogenesis decreases the rate of metastasis by $50-70 \% .{ }^{20}$ While angiogenesis in HCC was extensively analyzed and nowadays it is increasingly well understood, little is known about the role of lymphangiogenesis in this malignancy. In an HCC experimental model, Thelen et al. proved that lymphangiogenesis is involved in tumor growth and metastatic spread. ${ }^{21} \mathrm{In}$ addition, they demonstrated for the first time that LVD has a key role in tumor progression, tumor recurrence and survival in human HCC. ${ }^{22}$ By using D2-40 monoclonal antibody, we demonstrated intratumoral lymphatic vessels in $44.4 \%(32 / 72)$ of HCCs and peritumoral lymphatic vessels in
$79 \%(57 / 72)$ of our cases. Similarly to the Thelen et al. ${ }^{23}$ study, we showed that LVD is significantly associated with cirrhosis $(\mathrm{P}=0.006)$. In addition, our study demonstrated that high lymphatic density is correlated with vascular invasion $(\mathrm{P}=0.018)$, a parameter that typically predicts a more advanced tumor stage. Thus, we believe that tumor-associated lymphangiogenesis is involved in tumor growth, contributing to neovascularization and tumor invasion in human HCC.

Table 1. Relationship between lymphatic vessel density and clinicopathological parameters.

\begin{tabular}{|c|c|c|c|c|}
\hline Clinicopathological parameters & No. of cases & LVD peritumoral (Range) & LVD intratumoral (Range) & P-value \\
\hline $\begin{array}{l}\text { Gender } \\
\quad \text { Males } \\
\text { Females }\end{array}$ & $\begin{array}{l}50 \\
22\end{array}$ & $\begin{array}{l}14.92(0-45) \\
12.27(0-60)\end{array}$ & $\begin{array}{l}5.93(0-28) \\
5.61(0-24)\end{array}$ & $>0.05$ \\
\hline $\begin{array}{l}\text { Age } \\
\quad<60 \text { years } \\
>60 \text { years }\end{array}$ & $\begin{array}{l}21 \\
51 \\
\end{array}$ & $\begin{array}{l}14.19(0-35) \\
14.47(0-60)\end{array}$ & $\begin{array}{l}2.76(0-12) \\
6.94(0-28)\end{array}$ & $>0.05$ \\
\hline $\begin{array}{l}\text { Grade } \\
\text { Well differentiated (grade I) } \\
\text { Moderately differentiated (grade II) } \\
\text { Poorly differentiated (grades III, IV) }\end{array}$ & $\begin{array}{c}8 \\
30 \\
34\end{array}$ & $\begin{array}{l}8.62(0-28) \\
14.10(0-35) \\
15.73(0-60)\end{array}$ & $\begin{array}{l}5.37(0-28) \\
5.60(0-25) \\
6.14(0-25)\end{array}$ & $>0.05$ \\
\hline $\begin{array}{l}\text { Stage } \\
\text { I } \\
\text { II } \\
\text { III, IV }\end{array}$ & $\begin{array}{l}22 \\
26 \\
26\end{array}$ & $\begin{array}{l}13.72(0-29) \\
13.69(0-35) \\
13.56(0-60)\end{array}$ & $\begin{array}{l}8.04(0-28) \\
4.15(0-25) \\
5.11(0-20)\end{array}$ & $>0.05$ \\
\hline $\begin{array}{l}\text { Tumor size } \\
\qquad \begin{array}{l}<5 \mathrm{~cm} \\
>5 \mathrm{~cm}\end{array}\end{array}$ & $\begin{array}{l}46 \\
26\end{array}$ & $\begin{array}{l}16.30(0-45) \\
11.34(0-60)\end{array}$ & $\begin{array}{l}7.06(0-28) \\
4.57(0-20)\end{array}$ & $>0.05$ \\
\hline Vascular invasion & 25 & $15.32(0-60)$ & $3.60(0-20)$ & 0.018 \\
\hline Cirrhosis & 32 & $15.84(0-45)$ & $7.90(0-25)$ & 0.006 \\
\hline Chronic hepatitis & 19 & $11.21(0-29)$ & $5.00(0-28)$ & $>0.05$ \\
\hline
\end{tabular}

LVD, lymphatic vessel density.

Table 2. Relationship between podoplanin expression by tumor cells and clinicopathological parameters.

\begin{tabular}{|c|c|c|c|c|}
\hline \multirow[t]{2}{*}{ Clinicopathological parameters } & \multirow[t]{2}{*}{ No. of cases } & \multicolumn{2}{|c|}{ Podoplanin expression } & \multirow[t]{2}{*}{ P-value } \\
\hline & & Positive & Negative & \\
\hline \multicolumn{5}{|l|}{ Gender } \\
\hline Males & 50 & $15(30 \%)$ & $35(70 \%)$ & $>0.05$ \\
\hline Females & 22 & $7(32 \%)$ & $15(68 \%)$ & \\
\hline Age $<60$ years & 21 & $10(48 \%)$ & $11(52 \%)$ & $>0.05$ \\
\hline$>60$ years & 51 & $13(25 \%)$ & $38(75 \%)$ & \\
\hline \multicolumn{5}{|l|}{ Grade } \\
\hline Well differentiated (grade I) & 8 & $0(0 \%)$ & $8(100 \%)$ & 0.040 \\
\hline Moderately differentiated (grade II) & 30 & $9(30 \%)$ & $21(70 \%)$ & \\
\hline Poor differentiated (grades III, IV) & 34 & $14(41 \%)$ & $20(59 \%)$ & \\
\hline \multicolumn{5}{|l|}{ Stage } \\
\hline I & 22 & $7(32 \%)$ & $15(68 \%)$ & $>0.05$ \\
\hline II & 26 & $11(42 \%)$ & $15(58 \%)$ & \\
\hline III, IV & 26 & $5(19 \%)$ & $21(81 \%)$ & \\
\hline \multicolumn{5}{|l|}{ Tumor size } \\
\hline$<5 \mathrm{~cm}$ & 46 & $14(30 \%)$ & $32(70 \%)$ & $>0.05$ \\
\hline$>5 \mathrm{~cm}$ & 26 & $9(35 \%)$ & $17(65 \%)$ & \\
\hline Vascular invasion & 25 & $6(24 \%)$ & $19(76 \%)$ & $>0.05$ \\
\hline Cirrhosis & 32 & $13(41 \%)$ & $19(59 \%)$ & $>0.05$ \\
\hline Chronic hepatitis & 19 & $9(47 \%)$ & $10(53 \%)$ & $>0.05$ \\
\hline
\end{tabular}


Expression of podoplanin in tumor cells was found to play an important role in carcinogenesis, cell motility and tumor invasion. In addition, high podoplanin expression was strongly associated with an aggressive tumor behavior, with higher metastatic potential and with a poor survival in a wide variety of tumors. ${ }^{24-29}$ In the present study, we showed for the first time that podoplanin is expressed in the tumor cells of HCC and we found that high podoplanin expression is significantly correlated with a high histological grade $(\mathrm{P}=0.040)$, suggesting a role of podoplanin in hepatocarcinogenesis. Our findings are in good agreement with other studies that link podoplanin expression of tumor cells to histological grade in human cancers. ${ }^{23,30-32}$ Atsumi et al. demonstrated that podoplanin is a potential marker of tumor-initialing cells (TICs) with stem-cell-like properties in squamous cell carcinoma. ${ }^{33}$ In human HCC, cancer stem cells (CSCs) have been successfully identified and they are thought to be responsible for tumor recurrence and treatment failure. ${ }^{34-36}$ Kato et al. developed a cancer-specific $\mathrm{mAb}$ against podoplanin and demonstrated its effectiveness in an experimental model of glioblastoma. ${ }^{37}$ Thus, further studies targeting TICs in podoplanin-expressing HCCs and inhibiting podoplanin functions may lead to new antitumor strategies.

CAFs represent a major component of the tumor stroma and they have a pivotal role in cancer development. Since CAFs are assigned with various pro-tumoral roles, they are attractive and promising targets for cancer therapy. However, little is known about the role of CAFs in the human hepatocellular carcinoma microenvironment. Jia et al. demonstrated that CAFs are able to promote HCC proliferation and to support the growth of tumor cells. ${ }^{38}$ Moreover, their results show that CAFs support tumor cell survival in severe conditions, such as massive necrosis. Recent studies identified podoplanin as a marker of CAFs in various cancers and there is increasing evidence that its overexpression has a direct impact on both tumor growth and progression. ${ }^{14}$ In our study, podoplanin expression by CAFs was found in 41 cases (57\%) of HCC and it was significantly correlated with both LVD $(\mathrm{P}=0.019)$ and podoplanin-expressing tumor cells $(\mathrm{P}=0.015)$. It was already proved that podoplanin may mediate cancer cell migration. ${ }^{29,39}$ The most postulated theory indicate that cancer cells lose their epithelial phenotype and acquire a mesenchymal one, resulting in an increased migratory and invasive potential. ${ }^{31}$ To the best of our knowledge, the present study is the first to identify an interaction between epithelial and stromal tumor cells in HCC tumor microenvironment mediated by podoplanin. Further studies are necessary to clarify the biological functions and mechanism of podoplanin in HCC microenvironment.

In conclusion, our study shows the diversity of podoplanin biology in HCC. On the one hand, podoplanin proved to be a valuable marker in highlighting lymphatic vessel density. Our results suggest that tumor-associated lymphangiogenesis is involved in tumor growth, contributing to neovascularization and tumor invasion in HCC. Based on our results, we hypothesize that podoplanin might play a role in orchestrating the cross-talk between tumor cells and CAFs in human HCC microenvironment, although further studies are needed to elucidate this interrelation.

\section{References}

1. Chuma M, Terashita K, Sakamoto N. New molecularly targeted therapies against advanced hepatocellular carcinoma: From molecular pathogenesis to clinical trials and future directions. Hepatol Res 2015;45:E1-11.

2. Kaneko MK, Kato Y, Kitano T, Osawa M. Conservation of a platelet activating domain of Aggrus/podoplanin as a platelet aggregation-inducing factor. Gene 2006;378:52-7.

3. Kanner WA, Galgano MT, Atkins KA. Podoplanin expression in basal and myoepithelial cells: utility and potential pitfalls. Appl Immunohistochem Mol Morphol 2010;18:226-30.

4. Ordonez NG. Podoplanin: a novel diagnostic immunohistochemical marker. dv Anat Pathol 2006;13:83-8.

5. Jokinen CH, Dadras SS, Goldblum JR, van de Rijn M, West RB, Rubin BP. Diagnostic implications of podoplanin expression in peripheral nerve sheath neoplasms. Am J Clin Pathol 2008; 129:886-93.

6. Yu H, Gibson JA, Pinkus GS, Hornick JL. Podoplanin (D2-40) is a novel marker for follicular dendritic cell tumors. Am J Clin Pathol 2007;128: 776-82.

7. Suárez-Vilela Dimas, Izquierdo Francisco M. Podoplanin is positive not only on follicular dendritic cells and their tumoral counterparts, but also on reticular fibroblasts and in some tumors of reticular fibroblasts. Appl Immunohistochem Mol Morphol 2016;24:e11.

8. Breiteneder-Geleff S, Soleiman A, Kowalski H, Horvat R, Amann G, Kriehuber E, et al. Angiosarcomas express mixed endothelial phenotypes of blood and lymphatic capillaries: podoplanin as a specific marker for lymphatic endothelium. Am J Pathol 1999;154:385-94

9. Mishima K, Kato Y, Kaneko MK, Nakazawa Y, Kunita A, Fujita N, et al. Podoplanin expression in primary central nervous system germ cell tumors: a useful histological marker for the diagnosis of germinoma. Acta Neuropathol 2006;111:563-8.

10. Schacht V, Dadras SS, Johnson LA, Jackson DG, Hong YK, Detmar M. Upregulation of the lymphatic marker podoplanin, a mucin-type transmembrane glycoprotein, in human squamous cell carcinomas and germ cell tumors. Am J Pathol 2005;166:913-21.

11. Shibahara J, Kashima T, Kikuchi Y, Kunita A, Fukayama M. Podoplanin is expressed in subsets of tumors of the central nervous system. Virchows Arch 2006;448:493-9.

12. Raica M, Kondylis A, Mogoant L, Encică S, Cîmpean AM. Diagnostic and clinical significance of D2-40 expression in the normal human thymus and thymoma. Rom J Morphol Embryol 2010;51:229-34.

13. El-Gendi S, Abdel-Hadi M. Lymphatic vessel density as prognostic factor in br east carcinoma: relation to clinicopathologic parameters. J Egypt Natl Canc Inst 2009;21:139-49.

14. Pula B, Witkiewicz W, Dziegiel P, Podhorska-Okolow M. Significance of podoplanin expression in cancerassociated fibroblasts: a comprehensive review. Int J Oncol 2013;42:1849-57.

15. Suciu C, Muresan A, Cornea R, Suciu O, Dema A, Raica M. Semi-automated evaluation of $\mathrm{Ki}-67$ index in invasive ductal carcinoma of the breast. Oncol Lett 2014;7:107-14.

16. Gao J, Knutsen A, Arbman G, Carstensen J, Frånlund B, Sun XF. Clinical and biological significance of angiogenesis and lymphangiogenesis in colorectal cancer. Dig Liver Dig 2009;41:116-22.

17. Renyi-Vamos F, Tovari J, Fillinger J, Timar J, Paku S, Kenessey I, et al. Lymphangiogenesis correlates with lymph node metastasis, prognosis, and angiogenic phenotype in human nonsmall cell lung cancer. Clin Cancer Res 2005;11:7344-53.

18. Franchi A, Gallo O, Massi D, Baroni G, 
Santucci M. Tumor lymphangiogenesis in head and neck squamous cell carcinoma: a morphometric study with clinical correlations. Cancer 2004;101:973-8.

19. Zeng Y, Opeskin K, Horvath LG, Sutherland RL, Williams ED. Lymphatic vessel density and lymph node metastasis in prostate cancer. Prostate 2005;65:222-30.

20. Holopainen T, Bry M, Alitalo K, Saaristo A. Perspectives on lymphagiogenesis and angiogenesis in cancer. $\mathrm{J}$ Surg Oncol 2011;103:484-88.

21. Thelen A, Jonas S, Benckert C, Weichert W, Schott E, Bötcher C, et al. Tumor-associated lymphangiogenesis correlates with after resection of human hepatocellular carcinoma. Ann Surg Oncol 2009;16:1222-30.

22. Thelen A, Scholz A, Benckert C, von Marschall Z, Schröder M, Wiedenmann B, et al. VEGF-D promotes tumor growth andlymphatic spread in a mouse model of hepatocellular carcinoma. Int J Cancer 2008;122:247181.

23. Kadota K, Huang CL, Liu D, Nakashima N, Yokomise H, Ueno M, et al. The clinical significance of the tumor cell D2-40 immunoreactivity in non-small lung cancer. Lung Cancer 2010;70:88-93.

24. Tanaka M, Kijima H, Shimada H, Makuuchi H, Ozawa S, Inokuchi S. Expression of podoplanin and vimentin is correlated with prognosis in esophageal squamous cell carcinoma. Mol Med Rep 2015;12:4029-36.

25. Yuan P, Temam S, El-Naggar A, Zhou
X, Liu DD, Lee JJ, et al. Overexpression of podoplanin in oral cancer and its association with poor clinical outcome. Cancer 2006; 107:563-69.

26. Kato Y, Sasagawa I, Kaneko M, Osawa M, Fujita N, Tsuruo T. Aggrus: A diagnostic marker that distinguishes seminoma from embryonal carcinoma in testicular germ cell tumors. Oncogene 2004;23:8552-6.

27. Mishima K, Kato Y, Kaneko MK, Nishikawa R, Hirose T, Matsutani M. Increased expression of podoplanin in malignant astrocytic tumors as a novel molecular marker of malignant progression. Acta Neuropathol 2006; 111:483-8.

28. Abe S, Morita Y, Kaneko MK, Hanibuchi M, Tsujimoto Y, Goto $\mathrm{H}$, et al. A novel targeting therapy of malignant mesothelioma using antipodoplanin antibody. J Immunol 2013; 190:6239-49.

29. Takagi S, Oh-Hara T, Sata S, Gong B, Takami M, Fujita N. Expression of Aggrus/podoplanin in bladder cancer and its role in pulmonary metastasis. Int J Cancer 2014;134:2605-14.

30. Patil A, Patil K, Tupsakhare S, Gabhane $\mathrm{M}$, Sonune S, Kandalgaonkar S. Evaluation of podoplanin in oral leukoplakia and oral squamous cell carcinoma. Scientifica 2015;2015:135298.

31. Wicki A, Christofori G. The potential role of podoplanin in tumour invasion. Br J Cancer 2007;96:1-5.

32. Kim H, Rha K, Shim GA, Kim JH, Kim
JM, Huang SM, et al. Podoplanin is involved in the prognosis of head and neck squamous cell carcinoma through interaction with VEGF-C. Oncol Rep 2015;34:833-42.

33. Atsumi N, Ishii G, Kojima M, Sanada M, Fujii S, Ochiai A. Podoplanin, a novel marker of tumor-initiating cells in human squamous cell carcinoma A431. Biochem Biophys Res Commun 2008;373:36-41.

34. Chiba T, Kita K, Zheng YW, Yokosuka O, Saisho H, Iwama A, et al. Side population purified from hepatocellular carcinoma cells harbors cancer stem cellike properties. Hepatology 2006;44:240-51.

35. Ma S, Chan KW, Hu L, Lee TK, Wo JY, $\mathrm{Ng} \mathrm{IO}$, et al. Identification and characterization of tumorigenic liver cancer stem/progenitor cells. Gastroenterology 2007;132:2542-56.

36. Dean M, Fojo T, Bates S. Tumour stem cells and drug resistance. Nat Rev Cancer 2005;5:275-84.

37. Kato Y, Kaneko MK. A cancer-specific monoclonal antibody recognizes the aberrantly glycosylated podoplanin. Sci Rep 2014;4:5924.

38. Jia C-C, Wang T-T, Liu W, Fu BS, Hua $\mathrm{X}$, Wang GY, et al. Cancer-associated fibroblasts from hepatocellular carcinoma promote malignant cell proliferation by HGF secretion. PLoS One 2013;8: e63243.

39. Raica M, Cimpean AM, Ribatti D. The role of podoplanin in tumor progression and metastasis. Anticancer Res 2008; 28:2997-3006. 